\title{
Circ-ZNF609: a novel regulator of myogenesis
}

\author{
Paola Costelli ${ }^{1,2}$ \\ ${ }^{1}$ Department of Clinical and Biological Sciences, University of Torino, Torino, Italy; ${ }^{2}$ Istituto Interuniversitario di Miologia (IIM), Firenze, Italy \\ Provenance: This is a Guest Editorial commissioned by the Section Editor Jin Li (Cardiac Regeneration and Ageing Lab, School of Life Sciences, \\ Shanghai University, Shanghai, China). \\ Comment on: Legnini I, Di Timoteo G, Rossi F, et al. Circ-ZNF609 Is a Circular RNA that Can Be Translated and Functions in Myogenesis. Mol \\ Cell 2017;66:22-37.e9. \\ Correspondence to: Paola Costelli. Department of Clinical and Biological Sciences, University of Torino, Corso Raffaello 30,10125 Torino, Italy. \\ Email: paola.costelli@unito.it.
}

Received: 28 July 2017; Accepted: 02 August 2017; Published: 11 August 2017.

doi: $10.21037 /$ ncri.2017.08.01

View this article at: http://dx.doi.org/10.21037/ncri.2017.08.01

Skeletal muscle is the most abundant tissue in the body. It is composed by striated myofibers consisting of multinucleated syncytia deriving from proliferation and fusion of myogenic progenitor cells. As other terminally differentiated tissues, the skeletal muscle retains the ability to regenerate after damage, reactivating the myogenic process normally occurring in the embryonal and fetal life. Indeed, in postnatal skeletal muscle, a fraction of myogenic progenitors, namely satellite cells, remains quiescent in close proximity to myofiber basal membrane, ready to be reactivated in case of need. Despite such regenerative ability, however, altered muscle homeostasis is a frequent feature in both physiological (e.g., aging) and pathological states, such as muscle dystrophies, cancer, diabetes and neuromuscular diseases. As a result, the quality of life is severely compromised, rendering difficult to perform normal daily activities as well as impairing both tolerance and effectiveness of therapeutic regimens.

The most frequent alteration occurring in the skeletal muscle in aging as well as in different pathological conditions is a marked depletion of structural proteins that eventually leads to profound atrophy. The mechanisms underlying such wasting phenotype are not totally elucidated. Several studies have clearly demonstrated that protein turnover rates are poised towards a hypercatabolic setting, that can be associated or not with reduced protein synthesis. While this is a constant finding in muscle wasting, a number of reports have shown that also impaired myogenesis can play a role (1). This is quite obvious in pathologies associated with muscle damage, such as
Duchenne muscle dystrophy (DMD). It was a bit surprising to observe impairment in both myogenesis and regenerative response in aging-related sarcopenia and in pathologies that are not typically characterized by myofiber damage, such as diabetes and cancer (1).

Adult myogenesis is a tightly regulated process that is typically activated by myofiber injury. When this occurs, satellite cells are recruited to proliferate and differentiate in order to generate new myofibers or to fuse with the still existing ones, a process defined muscle regeneration. Such regenerative potential resembles the formation of multinucleated myofibers during the first steps of organogenesis and is associated with the timely expression of regulatory factors. Among these, Pax7 and myogenin show a sort of reciprocal regulation, the former being highly expressed in proliferating satellite cells, the latter being a marker of differentiation (2). In addition to satellite cells, other cell types can contribute to myogenesis, such as $\mathrm{PW}^{+}$/ $\mathrm{PAX}^{-}$interstitial cells (PICs) or mesoangioblasts (1).

While the broad regulation of myogenesis is well known, the mechanisms underlying its activation and completion, especially in pathological states, are not totally clear. In this regard, evidence has been provided that noncoding RNAs, in particular microRNAs (miRs) and long noncoding RNAs (lncRNAs), contribute to myogenesis as well as to the acquisition of muscle function. Discovered in the last decade of the past century, miRs are short noncoding RNAs that regulate gene expression targeting the 3' untranslated region (UTR) of mRNAs, resulting in accelerated degradation of mRNA and/or inhibition of translation. MiRs have 
been involved in several events such as cell proliferation, differentiation and death, DNA repair, oxidative stress response. Specific miRs have been indicated as musclespecific, or myomiRs, in view of their involvement in different steps of myogenesis and/or regulation of muscle trophism. As for lncRNAs, they are about 10 folds larger than miRs (about 200 and 20 nucleotides, respectively), do not code for proteins, and can be polyadenylated. They have been proposed to regulate gene expression, even if the underlying mechanisms are still poorly defined (3).

A recent study by Legnini and collaborators (4) proposes that also circular RNAs (circRNAs) can participate in the regulation of myogenesis. In particular, they identify circ-ZNF609 as able to specifically modulate myoblast proliferation.

CircRNAs are RNA molecules that lack both the 5' and 3' ends, structured as covalently closed continuous loops, usually generated by a back-splicing reaction. They are expressed by a high number of genes and appear to be highly conserved among species. Recently circRNAs have been involved in human fetal development, myocardial infarction and carcinogenesis. Little is known about the mechanism of action of circRNAs. Some studies have shown that they can act as miR sponges and a circRNAmiR-mRNA axis has been proposed. CircRNAs can also participate in transcriptional regulation, in competition with linear splicing and operate protein sequestration in the cytoplasm. Finally, circRNAs with open reading frames can be translated giving rise to peptides (5).

The study by Legnini et al. (4) describes for the first time the circRNA profile of human and murine myocytes, both proliferating (myoblasts) and after differentiation to myotubes. As a first step, the authors performed a RNA sequencing (RNA-seq) analysis, revealing that the pattern of gene expression in myoblasts is markedly different from that of myotubes; in particular, genes upregulated in myotubes pertain to pathways associated with myogenic differentiation and muscle function. The authors validated by qRT-PCR four myogenic markers, namely MyoD, myosin heavy chain, muscle creatine kinase and dystrophin. Searching for circRNA, Legnini et al. (4) observed that they were highly frequent and significantly conserved between the two species analyzed (mice and human beings). About $90 \%$ of circRNA derived from internal exons of protein coding genes, and $10 \%$ where expressed more than their linear counterpart. A linear correlation is reported between circRNAs and linear RNAs. Similar to total RNA-seq, also the expression of circRNA is quite different in myoblasts $v s$. myotubes.
The authors also report a peculiar circRNA profile in myoblasts and myotubes. In particular, the abundance of circRNAs seems to increase in differentiated $v s$. nondifferentiated cells. The same pattern can be observed for the circular/linear RNA ratio, likely due to both the increased circRNA transcription rate and to its higher stability in comparison to linear mRNAs. CircRNA profile in myoblasts and myotubes derived from DMD patients is also different from that observed in normal cultures.

To have information about the role of some of the differentially expressed circRNAs, the authors adopted a RNA interference-based functional screening. They identified circ-QKI as positive regulator of myogenic differentiation, a function that is shared with the linear QKI mRNA. By contrast, circ-BNC2 appeared to stimulate myogenesis by competing with the production of the respective linear mRNA. In other words, high circ-BNC2 levels were associated with low linear BNC2 mRNA, suggesting that the role of the former is to reduce the production of the latter, antagonizing its anti-myogenic potential. Both circRNAs, whose expression is increased in normal myotubes $v s$. myoblasts, favoring differentiation, are down-regulated in DMD cultures. This observation is consistent with the wellknown impairment of myogenic differentiation occurring in the skeletal muscle of DMD patients.

Legnini and collaborators (4) observed an opposite function for circ-ZNF609. It was highly expressed in myoblasts, and its down-regulation by specific siRNAs resulted in marked inhibition of myoblast proliferation, evaluated as reduced $\mathrm{BrdU}$ incorporation and decreased expression of CDK1 and cyclinA2. Circ-ZNF609 was highly expressed in DMD myocyte cultures, consistently with the impaired differentiation displayed by these cells. These results suggest the occurrence of a reciprocal regulation of circ-QIK/circ-BCN2 and circ-ZNF609, the former stimulating myogenic differentiation, the latter muscle progenitor expansion. However, different from circ-QIK and circ-BNC2, whose bioactivity derives from cooperation (circ-QIK) or antagonism (circ-BNC2) with their linear counterpart, circ-ZNF609 acts specifically on myoblast proliferation, without interfering with the linear mRNA.

Since circ-ZNF609 contains a 753-nucleotide open reading frame, the authors investigated if it could be translated into a protein. The results show that circ-ZNF609 can interact with heavy polysomes and that it can be effectively translated into a protein, even if the efficiency of translation is markedly lower than that displayed by the linear mRNA counterpart. CircRNAs do not have a cap 
structure, so they can only be translated in a cap-independent manner. Consistently, the UTR of circ-ZNF609 acts as an internal ribosome entry site (IRES) in a splicing-dependent manner. Cap-independent translation is often up-regulated in condition of cell stress, to achieve rapid modulations of protein expression. Indeed, the results reported by Legnini et al. (4) show that circ-ZNF609 translation can be up-regulated by heat shock. Finally, circ-ZNF609 in myoblasts displays a high degree of methylation, suggesting an additional mechanism of translational regulation.

At present the function of the protein encoded by circZNF609 is totally unknown. It might well be endowed with independent activity or it can be important to modulate the protein produced translating the linear mRNA. This latter protein is a transcription factor containing two zincfinger domains that has been reported to directly target the Rag 1 promoter in $\mathrm{T}$ lymphocyte precursors during thymic maturation. In this regard, mutations affecting ZNF609 may produce immunodeficiency or autoimmune response (6). ZNF609 has also been shown to directly interact with Dax1 (7) and with Nipbl (8), contributing to the maintenance of embryonic stem cell pluripotency or regulating neuronal migration genes, respectively. Legnini and collaborators (4) show that the protein encoded by circZNF609 lacks the zinc-finger domains, suggesting that it could be a dominant-negative competitor of the native protein, or become an alternative partner in the ZNF609 complex formation with other proteins (see above).

The study by Legnini et al. (4) proposes for the first time that circ-ZNF609 might exert a regulatory action by encoding a protein. Indeed, the literature reports just one study showing that circ-ZNF609 regulates AKT3 expression in Hirschsprung disease acting as a sponge for miR-150-5p, thus behaving as an endogenous competitor (9). In addition, Legnini and collaborators (4) propose a novel mechanism regulating myogenesis, in both physiological and pathological states. At present, there are no effective treatments able to interfere with the development of muscle wasting. In this regard, understanding the mechanisms of myogenesis and muscle function will prove valuable for the comprehension of muscle-associated diseases as well as for the design of appropriate therapeutic strategies. The new findings by Legnini et al. (4) perfectly fit with this picture, providing cues useful to set up targeted interventions responding to the concept of both regenerative and precision medicine.

\section{Acknowledgements}

None.

\section{Footnote}

Conflicts of Interest: The author has no conflicts of interest to declare.

\section{References}

1. Costamagna D, Costelli P, Sampaolesi M, et al. Role of Inflammation in Muscle Homeostasis and Myogenesis. Mediators Inflamm 2015;2015:805172.

2. Chal J, Pourquié O. Making muscle: skeletal myogenesis in vivo and in vitro. Development 2017;144:2104-22.

3. Simionescu-Bankston A, Kumar A. Noncoding RNAs in the regulation of skeletal muscle biology in health and disease. J Mol Med (Berl) 2016;94:853-66.

4. Legnini I, Di Timoteo G, Rossi F, et al. Circ-ZNF609 Is a Circular RNA that Can Be Translated and Functions in Myogenesis. Mol Cell 2017;66:22-37.e9.

5. Rong D, Sun $\mathrm{H}, \mathrm{Li} Z$, et al. An emerging function of circRNA-miRNAs-mRNA axis in human diseases. Oncotarget 2017. [Epub ahead of print]

6. Reed NP, Henderson MA, Oltz EM, et al. Reciprocal regulation of Rag expression in thymocytes by the zincfinger proteins, Zfp608 and Zfp609. Genes Immun 2013;14:7-12.

7. Wang J, Rao $\mathrm{S}$, Chu J, et al. A protein interaction network for pluripotency of embryonic stem cells. Nature 2006;444:364-8.

8. van den Berg DL, Azzarelli R, Oishi K, et al. Nipbl Interacts with Zfp609 and the Integrator Complex to Regulate Cortical Neuron Migration. Neuron 2017;93:348-61.

9. Peng L, Chen G, Zhu Z, et al. Circular RNA ZNF609 functions as a competitive endogenous RNA to regulate AKT3 expression by sponging miR-150-5p in Hirschsprung's disease. Oncotarget 2017;8:808-18. doi: 10.21037/ncri.2017.08.01

Cite this article as: Costelli P. Circ-ZNF609: a novel regulator of myogenesis. Non-coding RNA Investig 2017;1:2. 